\title{
Análisis de la bibliografía existente sobre inmigrante, actividad física, deporte e integración en lengua inglesa
}

\author{
Jesús Fernández Gavira* \\ Francis Ries \\ $M^{a}$ Ángeles Huete García \\ Jerónimo García Fernández
}

\begin{abstract}
Resumen: El artículo que se expone es una revisión de la literatura en inglés existente sobre integración, colectivos de inmigrantes, actividad física y deporte. La bibliografía encontrada se obtuvo de bases de datos internacionales usando varias combinaciones de descriptores. Los estudios fueron clasificados según los criterios de cronología, idioma y áreas de estudio. De la información obtenida con los 50 artículos se concluye que el campo científico del deporte y la inmigración es muy heterogéneo, siendo la migración de talentos deportivos y los estudios sociológicos relacionados con el deporte y la inmigración, las temáticas más repetidas.
\end{abstract}

Palabras clave: Emigrantes e Inmigrantes. Valores sociales. Deportes. Revisión.

\section{INTRODUCCIÓN}

Los espacios deportivos para las personas inmigrantes constituyen lugares de encuentro donde no solo se practica actividad física, sino que en ellos además se convive y se intercambia información valiosa sobre aspectos prácticos de la vida cotidiana. Estos espacios de encuentro multiculturales y lo que en ellos ocurre en referencia al deporte están siendo estudiados desde hace décadas en Europa y otras partes del mundo. No obstante gran parte de la información que se genera en esta dimensión se pierde, ya que la mayoría de los proyectos que se llevan a cabo se realizan desde

\footnotetext{
"Departamento de Educación Física. Universidad de Sevilla. Sevilla, España. E-mail: jesusfgavira@us.es
} 
entidades no académicas que no conocen la importancia o no son incentivadas a compartir los conocimientos que ellas generan de la puesta en práctica de sus intervenciones respecto a lo cual poco o nada se puede hacer.

No obstante y de cara a plantear una sólida base para futuros estudios se plantea como objetivo del presente el recabar la máxima información sobre los artículos escritos relacionados con la integración social, deporte, actividad física e inmigración indexados en bases de datos científicas de reconocido prestigio.

\section{Metodología}

La búsqueda se ha realizado en las principales bases de datos socio deportivas SCOPUS and Sportdiscus (EBSCO) utilizando los términos en inglés: migrant, sport, physical activity and integration, cruzándolos con el operador booleano AND y eliminando los artículos que se repetían. Al final en nuestra selección de estudios quedaron solamente aquellos relacionados directamente con el tema en cuestión.

Efectuada la búsqueda se establecieron varios criterios de inclusión para seleccionar los artículos, se presentan como sigue: artículos comprendidos entre 2000 y 2011; artículos escritos completa o parcialmente (resumen) en inglés; artículos de las áreas sociología y actividad física y deporte (cuando las bases de datos permitían la elección). Con el fin de encontrar los artículos relevantes, se hicieron un total de 7 búsquedas exhaustivas en las bases de datos Scopus y Sportdiscus, utilizando para ello los siguientes descriptores: sport, values and migration; sport, values and integration; migration and Sport; migration, sport and integration; migration and physical activity; migration, physical activity and integration; sport migration. Todos estos elementos enumerados anteriormente fueron incorporados en las sucesivas pesquisas de manera sistemática con la finalidad de obtener resultados objetivos y repetibles. Las búsquedas comenzaron el uno de julio de 2011 y finalizó el tres de noviembre de 2011. Para el análisis de los resultados obtenidos, se diseñó una base de datos en el programa informático Microsoft Excel 2010. La extracción de 
los datos la realizaron tres investigadores independientes de las universidades de Sevilla y Pablo de Olavide del ámbito de la sociología y ciencias del deporte con experiencia en la temática, a los que se les dieron las consignas anteriores. Todos efectuaron la búsqueda de manera separada y posteriormente se puso en común la información obtenida.

\section{Resultados Y discusión}

La búsqueda arrojó un total de 649 resultados, de los que se seleccionaron 72 (Tabla 1) por cumplir los criterios antes señalados (cronología, idioma y áreas de estudio). Los cuales a su vez al verse algunos de ellos repetidos en las dos bases de datos, se redujeron a 50.

Tabla 1: Número de artículos según descriptores

\begin{tabular}{|c|c|c|c|}
\hline Bases de datos & Descriptor & Resultados & $\begin{array}{l}\text { Artículos } \\
\text { Selecciona dos }\end{array}$ \\
\hline Scopus ( $1^{\mathrm{a}} \mathrm{B}$ úsqueda) & \multirow{2}{*}{ Sport, values and migration } & 16 & 0 \\
\hline Sportdiscus ( $1^{\mathrm{a}}$ Búsqueda) & & 33 & 15 \\
\hline Scopus ( $2^{\mathrm{a}} \mathrm{B}$ úsqueda) & \multirow{2}{*}{ Sport, values and integration } & 45 & 3 \\
\hline Sportdiscus ( $2^{\mathrm{a}}$ Búsqueda) & & 46 & 3 \\
\hline Scopus ( $3^{\mathrm{a}}$ Búsqueda) & \multirow{2}{*}{ Migration and Sport } & 222 & 17 \\
\hline Sportdiscus ( $3^{\mathrm{a}}$ Búsqueda) & & 55 & 12 \\
\hline Scopus ( $4^{\mathrm{a}} \mathrm{B}$ úsqueda) & \multirow{2}{*}{ Migration, Sport and Integration } & 14 & 1 \\
\hline Sportdiscus (4 $4^{\mathrm{a}}$ Búsqueda) & & 3 & 2 \\
\hline Scopus (5 Búsqueda) & \multirow{2}{*}{ Migration and Physical Activity } & 63 & 2 \\
\hline Sportdiscus ( $5^{\mathrm{a}}$ Búsqueda) & & 7 & 1 \\
\hline Scopus ( $6^{\mathrm{a}}$ Búsqueda) & \multirow{2}{*}{$\begin{array}{l}\text { Migration, Physical Activity } \\
\text { and Integration }\end{array}$} & 13 & 1 \\
\hline Sportdiscus ( $6^{\mathrm{a}}$ Búsqueda) & & 34 & 3 \\
\hline Scopus ( $7^{a}$ Búsqueda) & \multirow{2}{*}{ Sport Migration } & 76 & 10 \\
\hline Sportdiscus ( $7^{\mathrm{a}}$ Búsqueda) & & 22 & 2 \\
\hline \multicolumn{2}{|l|}{ Artículos totales } & 649 & 72 \\
\hline \multicolumn{2}{|l|}{ Artículos Repetidos } & 22 & \\
\hline
\end{tabular}

Fonte: de los auctores

Estos 50 artículos a su vez fueron analizados detenidamente y clasificados según su contenido en seis áreas temáticas diferenciadas en función de su naturaleza. (Ver tabla 2): 
Tabla 2: Áreas temáticas de estudio

\begin{tabular}{lc}
\hline ÁREAS TEMÁTICAS & $\begin{array}{l}\text { ARTÍCULOS } \\
\text { SELECIONADOS }\end{array}$ \\
\hline Género, deporte e inmigración & 6 \\
Estudios históricos, deporte e inmigración & 3 \\
Deporte profesional y migración de los talentos deportivos & 18 \\
Otros estudios sociológicos relacionados con el deporte y la & 16 \\
inmigración & 5 \\
Estudios en relación a deporte e inmigración que tienen lugar en & \\
espacios físicos concretos & 2 \\
Legislación sobre deporte e inmigración & \\
\hline
\end{tabular}

Fonte: de los auctores

En relación al género, deporte e inmigración, Yang (2007) muestra como una comunidad de mujeres chinas residentes en Vancouver a través de la danza china como actividad tradicional generan un espacio de encuentro que las dota de identidad de grupo mientras realizan actividad física con beneficios para su salud. En una línea parecida Pfister (2000) y Eun-Ok E.-O y Myoung-Ae (2001) ilustran casos parecidos, la primera el de un grupo de mujeres turcas de segunda generación residentes en Alemania que realizan actividades físicas de mantenimiento a través de la asociación nacional de clubes deportivos (FDA), y los segundos el caso de 54 mujeres coreanas residentes en USA, sin experiencia en la práctica deportiva que entraron en el sistema público a través de programas específicos para ellas.

En otra línea Agergaard (2008), profundiza en el caso de las mujeres extranjeras deportistas de alto nivel que participaron en la liga de balonmano de Dinamarca desde 1999 a 2007, así como los motivos que las llevaron a ir a Dinamarca y participar de las ligas nacionales de balonmano. Este caso que también podría incluirse en el punto de migración por motivos laborales, es a su vez un buen ejemplo de estudio específico sobre el colectivo femenino.

El artículo de Hibbins (2006) es especialmente interesante en el sentido que trata al deporte en relación al género masculino, 
estudiando el caso de una pequeña comunidad china de un municipio australiano. Esta población tiene muchas creencias machistas que se reflejan en la vida de estas personas, entre estas se incluyen, que los hombres sean los proveedores de comida y protectores de la familia.

En relación a los estudios históricos, deporte e inmigración, dos de ellos se refirieron a población migrada entre zonas próximas durante un periodo concreto del siglo XX. Son los casos de Sudáfrica y Polonia - Alemania, y de cómo el boxeo y el fútbol facilitaron los procesos de integración en los lugares donde llegaron.

De esta manera Fleming (2011) profundiza en el crecimiento exponencial que el boxeo tiene en la población sudafricana emigrada a Witwatersrand (Johannesburgo), el cual aunque en un primer momento fue empleado como una herramienta para la supervivencia en su nuevo entorno, pronto es reconducido y adquiere para la población que lo practica una serie de ideales entre los que se incluyen: la autodisciplina, civismo e independencia.

Otro ejemplo de integración entre poblaciones relativamente cercanas, es el de Lenz (2006) que muestra el proceso de integración vivido por la población polaca y de Masur, tras emigrar a principios del siglo pasado a la región de Ruhr de Alemania, la cual al principio se observó desde la población local con resentimiento y como amenaza, a lo que la población emigrada contestó cerrándose y creando sus propias comunidades étnicas, en las que desarrollaban su propia cultura de origen. En este marco de segregación, la creación de los primeros clubes de fútbol de inmigrantes que participaban en las ligas alemanas facilitó el contacto y por lo tanto la integración entre autóctonos e inmigrantes recién llegados, lo cual se convertiría en un hecho con el nacimiento y participación de las segundas generaciones de inmigrantes en estos campeonatos.

Finalmente otro trabajo que requiere la atención en esta área es el de Taylor (2006), donde se analiza el fenómeno migratorio en 
relación al mercado laboral deportivo, concretamente el fútbol, pero desde una perspectiva histórica, retrotrayéndose a los años $30 \mathrm{del}$ pasado siglo hasta llegar al año 2000.

Con respecto a los estudios de deporte profesional y migración de los talentos deportivos casi todos siguen una línea argumental común que es analizar la migración de los talentos deportivos desde unos países de origen a otros de destino, analizando los procesos personales pasados por los deportistas cuando emigran, el sentimiento que en ellos se genera, la repercusiones que tienen cuando consiguen éxito en su carrera deportiva, los cambios que la migración de estos jugadores tiene en su propia comunidad de destino o de origen, y la manera en que el estilo de vida del jugador o sus propios valores se ven influenciados durante el transcurso de su vida deportiva.

En relación a los procesos personales vividos por los deportistas Carter (2007) indaga en el impacto que se genera en el núcleo familiar cuando uno de sus miembros falta. De manera particular presenta el estudio de casos de dos familias en el que uno de sus miembros emigra por motivos profesionales (deportivos) acompañándolos en su hégira deportiva.

Profundizando en las grandes ligas europeas y americanas de fútbol y baloncesto actúan como foco de atención y referente de las personas emigrantes, Collegue (2010) dice de ellas que son también lugares de consumo y producción de jugadores profesionales en las que hay una gran movilidad de los mismos. En este sentido en el baloncesto más que ningún otro deporte, el número de extranjeros en ligas profesionales es el más elevado, superándose en gran parte de esos equipos el número de extranjeros frente al de autóctonos. En relación al fútbol profesional, Poli (2007) afirma que los inmigrantes latinos y africanos llegan a representar el $20 \%$ del total de la plantilla de los mismos y alude además al igual que Maguirre y Pearton (2000), que las razones por las que unos jugadores elijen emigrar a unos países frente a otros son históricas, políticas y lingüísticas y no tanto económicas. 
En esta línea, en el caso de ligas profesionales de beisbol del pacífico y norteamericanas (CHIBA, 2004; TAKANASI; HOME, 2006) se pone de manifiesto como los jugadores extranjeros proceden en mayor medida de América Latina, a razón del $90 \%$ en Estados Unidos y $50 \%$ en las ligas del pacífico asiático, habiendo un intercambio de jugadores entre ambas.

Autores como Cornelissen y Solberg (2007) afirman que algunos países emergentes dentro de los continentes emisores de emigrantes por motivos laborales, son en la actualidad los nuevos focos hacia los que se dirigen una parte de los flujos migratorios, así por ejemplo Sudáfrica empieza a emerger como uno de los nuevos lugares donde hacer carrera futbolística en el que quedarse.

Muy interesante resulta la perspectiva desde la que Vasconcellos y Dimeo (2009) estudian la emigración de jugadores, en su caso indagan en lo que significa para un grupo de familias brasileñas que uno de sus miembros jóvenes se vaya, de la misma manera se hace un seguimiento de los mismos con el objetivo de estudiar cómo va evolucionando su identidad en el extranjero, la cual se forja en contacto con la población local que en muchos casos y sobre todo en Europa resultan muy cerrada al contacto con el emigrante. En el caso del África sub-sahariana Poli (2006) ser futbolistas de alto nivel supone representar en sus países de origen una figura de poder a tres niveles, económico, político (embajador del país) y social (mediador de conflictos en problemas), por lo que la persona que lo consigue se convierte en icono de prestigio muy deseable. Esto resulta tentador para muchísimos jóvenes que salen de sus países de origen en pos de sueño difícilmente alcanzable (POLI, 2010b).

Desde la perspectiva de la gestión del poder y la influencia que ejercen como figuras mediáticas los jugadores emigrados desde las ligas profesionales de alto nivel (estadounidenses) sobre los hábitos de vida de la población local de las ciudades inglesas donde llegan para incorporarse a los nuevos equipos, lo estudian Maguirre (2004) y Falcous y Maguirre. (2005). Lo cual se hace evidente en la americanización de sus seguidores, observable en sus vestimentas y hábitos de consumo. 
En la investigación sobre talentos deportivos y las consecuencias morales y económicas que supone el traslado de estas personas desde unos países y otros, investigan autores como Cameron (2000), Lopes y Waddinton (2004) y Darvy, Arkindes y Kirwis (2007).

De manera específica Cameron (2000) analiza la migración desde Nueva Zelanda a Estados Unidos, país que dota a estas personas de becas deportivas. Lo cual presenta una problemática, en el sentido de que la inversión en el entrenamiento de esas personas se realiza a cargo de los clubes de origen y cuando ya son promesas, hay un elevado número de jóvenes que migran al continente americano, suponiendo para sus clubes de origen pérdidas deportivas y económicas.

Desde el punto de vista político y en línea con el punto anterior, Maguirre (2008) profundiza en los procesos vividos por estas personas para tomar la decisión de emigrar y lo que significa para el país de origen la salida de sus talentos al exterior, también se reflexiona en la acogida por parte de los países de destino de estos deportistas, los cuales a veces se ven obligados a crear nuevas políticas migratorias para dar cabida a nuevos fenómenos que se producen alrededor del deporte.

Otros autores por su parte deciden estudiar concretamente el deporte dentro de un sistema global como ejemplo de microsistema, en el que se puede observar todos los fenómenos que se dan en el resto de la sociedad actual, así Poli (2010a) analiza la globalización y el nuevo sistema laboral que de ella emerge a través del fútbol, el cual resulta un buen ejemplo donde explicar la nueva división del trabajo y los flujos migratorios.

En relación con la temática Otros estudios sociológicos relacionados con el deporte y la inmigración, algunos profundizan en las diferentes estrategias políticas que se sirven del deporte como medio para poner en comunicación a inmigrantes y autóctonos, otros indagan en la identidad transnacional de los emigrados y la identificación de los mismos con sus equipos de fútbol más que con sus propios países, dando lugar a identidades híbridas, otros investigan en la significación propia del deporte, sus canciones 
y símbolos en relación a la población autóctona y minorías étnicas, a continuación se irán desglosando las áreas identificadas.

En relación a si el grado de aculturación aumenta o disminuye con la práctica deportiva durante el tiempo libre de los turcos residentes en los Países Bajos, Hosper, Klazinga y Stronks (2007) manifiestan que esta relación solo se da de manera directa con respecto al nivel adquisitivo, de manera que a mayores ingresos también aumenta la práctica deportiva de esta población. Por otra parte no se encuentran relaciones entre tener niños, trabajar o vivir en barrios socialmente desfavorecidos con realizar más o menos práctica deportiva en el tiempo de ocio. Con respecto a estos tres últimos parámetros, Marlière (2008) indaga en las prácticas culturales y deportivas de los jóvenes inmigrantes de barriadas deprimidas, las cuales pueden ser diferentes en función de la edad de los mismos, curso escolar, afinidades u origen étnico.

Otro de los temas recurrentes sobre la temática son los estudios sobre las aficiones de los equipos de fútbol (MONIZ, 2007; SCHOONDERWOERD, 2011; TAINSKY; STODOLSKA, 2010).

Tainsky y Stodolska (2010) se interesan por los seguidores de los equipos locales cuando estos emigran de unas ciudades a otras del país con el objetivo de averiguar con qué equipo se identificaban entonces, resultando que la población emigrada sigue apoyando sobre todo en los primeros momentos a los antiguos, independientemente de que los equipos actuales tuvieran una mejor proyección en la liga. Esto ocurrió en mayor medida en las ciudades donde la población tenía menor renta per cápita, relacionándose de esta manera menores recursos económicos con mayor lealtad con los equipos de origen.

Moniz (2007) analiza las aficiones de los inmigrantes pero desde una perspectiva transnacional. Así focaliza su atención en la población lusófona residente en Estados Unidos y cómo esta se identifica con el equipo de fútbol de Nueva Inglaterra que juega en la primera liga nacional, y que representa los valores y el éxito de una población emigrante. 
Por su parte Schoonderwoerd (2011) analiza las canciones de las aficiones de los equipos de fútbol europeos desde sus contenidos, con el objetivo de identificar señales de identidad tanto individuales como colectivas en la historia reciente de este deporte, la cual a menudo se relaciona con la historia de los orígenes de los equipos y de sus seguidores.

En relación al deporte, inmigración y política, Dietschy (2006) analiza el uso del deporte como estrategia política a través de diferentes experiencias recogidas a lo largo de la historia del fútbol moderno y que vienen recogidas en la base de datos de la FIFA. Entre los casos seleccionados, estarían los equipos vascos de fútbol que apoyaron al bando republicano durante la guerra civil española e hicieron una travesía por América latina mezclando deporte con arengas políticas, o en Italia donde el orgullo patrio durante el gobierno de Mussolini se hizo especialmente manifiesto en su equipo nacional de fútbol que representó la sangre italiana dentro y fuera del país.

Desde una perspectiva diferente a lo visto hasta ahora, Humphreys y Munich (2008) profundizan en la economía como principal responsable de migración forzada de algunos deportistas desde sus países de origen con menos recursos a otros enriquecidos, y como la enseñanza de entrenamientos específicos de los países de destino, ayudan a disminuir la deuda de los países de origen en forma de subvenciones internas al deporte local.

Ahondando más en la responsabilidad de la globalización en los procesos de asimilación, Dimeo y Vasconcellos (2009) presentan un estudio de investigación sobre jugadores profesionales brasileños de futsal, en relación a su experiencia social y deportiva e identidad nacional como inmigrados en España y Portugal, los cuales, al ser entrevistados de regreso a sus países, en su mayoría coincidieron en que tuvieron una experiencia positiva, de manera particular por haber adquirido un estilo europeo de jugar, a la vez que gran desarraigo con su país con respecto al cual estaban dispuestos a renunciar a su identidad brasileña con la condición de jugar en la primera liga nacional de los países en los que habían estado. 
Terret (2008) analiza los aspectos socio culturales, socio afectivos y estructurales que se ven facilitados por la práctica deportiva de cara a la integración social, detectando que el racismo, el género y la competitividad son tres de los factores que impiden la integración.

De manera más local, Sine y Sorensen (2010) buscan en el sistema público deportivo danés ejemplos de buenas prácticas deportivas donde se dé la integración social. De esta manera estudian cómo el gobierno delega la realización de programas deportivos en las redes nacionales de clubes deportivos a los que financia para ello, utilizando varias estrategias, entre las que están en un primer momento la multiculturalidad que se observa al respetar y permitir que cada jugador use la vestimenta y coma lo que les parezca adecuado, mientras que en otras circunstancias imponen las normativas del grupo mayoritario tales como horarios y días de entrenamiento, estrategia más específica de la asimilación.

Otros autores Müler, Van Zoonen y De Roode (2008) analizan los eventos deportivos multiculturales buscando ejemplos de integración. El análisis de los autores al respecto, arroja entre otros resultados que los campeonatos investigados por ellos y por inducción de la mayoría, no fueron eficaces en relación a la construcción de relaciones sociales entre los participantes.

Más específicamente Arnaud (2002) realiza un estudio comparativo entre dos ciudades europeas, Lyon (Francia) y Birmingham (Reino Unido). Observándose que en ambos casos las minorías étnicas intentaban organizar sus propios eventos deportivos contando con la ayuda económica de los gobiernos locales, en los que solían darse aquellas actividades que ya se hubieran demostrado como válidas haciéndose muy rutinarias.

Existen ciertos países con un gran arraigo cultural que a pesar de ser tradicionalmente emisores de emigrantes mantienen sus costumbres culturales y deportivas dentro y fuera del país. Es el caso de los irlandeses en el exterior como exponen Cronin, Doyle y O'Callaghan (2008), donde los emigrados no han sido absorbidos en 
materia deportiva por estrategias asimilacionistas de los países de destino, muy al contrario sus comunidades en el exterior han exportado el deporte nacional como un signo que les dotaba de identidad como el hurling o el fútbol gaélico. No obstante, dentro de sus fronteras han irrumpido nuevos deportes con fuerza como el Rugby inglés que convive con los deportes tradicionales irlandeses en mutuo beneficio.

Otros países sin deportes autóctonos representativos, pero con idéntica necesidad de identidad nacional en el extranjero, buscan incluirse en el sistema nacional deportivo del país receptor en un proceso de etnificación comunitario, constituyéndose en equipos de su misma nacionalidad. Así por ejemplo Blecking (2008) trata el caso de los inmigrantes polacos antes de la primera guerra mundial en Alemania. El mismo fenómeno ocurriría 50 años más tarde con la llegada de los turcos al territorio alemán, los cuales se establecieron de manera permanente en las ligas alemanas de fútbol, llegándose a la conclusión de que el deporte en ambos casos cumplió el mismo objetivo, la creación de su propia identidad étnica en el nuevo territorio.

En relación a los Estudios en relación a deporte e inmigración que tienen lugar en espacios físicos concretos destacan algunos autores como Urniaz (2006) y Sugden (2006). Así por ejemplo una línea consolidada es la de los campeonatos deportivos realizados en lugares transfronterizos entre diferentes países limítrofes con el objetivo de observar de qué manera el deporte podría ser un posible elemento conciliador entre culturas. Así ocurre según Urniaz (2006) en las regiones de Warmia- Mazury (Polonia) y Kaliningrado (Rusia), las cuales si bien tradicionalmente han estado en conflicto continuo, han encontrado en el deporte una herramienta de unión que les sirve de excusa para establecer relaciones de igualdad más profundas entre ellos. De esta manera los residentes de una y otra zona cruzan la frontera no tanto con el objetivo de obtener resultados deportivos sino más en la línea seguir estrechando relaciones personales con sus vecinos y divertirse. 
En la misma línea Sugden (2006) hace un estudio descriptivo y crítico de la situación de un proyecto deportivo (futbolístico) que tiene lugar en Israel entre las comunidades árabes y judías, con el objetivo de favorecer el contacto entre las personas, en aras de favorecer el entendimiento y la paz entre las mismas, utilizando como principales valores, el respeto, compañerismo, igualdad y responsabilidad. Los primeros resultados de la investigación determinaron que el proyecto "Fútbol para la paz" resultó eficaz para la consecución de los objetivos propuestos, si bien hay que trabajar en la inclusión de las mujeres que han sido las grandes ausentes en estos espacios.

En relación a los programas deportivos para jóvenes de diferentes minorías étnicas escolarizados en Canadá, Alison y Traylor (2007) les preguntaron por su percepción sobre los mismos, mostrándose en la mayoría de los casos un acogimiento positivo hacia estos programas. No obstante una parte representativa de esta población no encuentra adaptadas dichas iniciativas debido a una falta de conocimiento del idioma local, costumbres culturales de género, o prejuicios personales o de grupo.

Otra línea muy interesante es el estudio de los inconvenientes para la práctica deportiva que exponen Tcha y Lobo (2003) que estudian el caso la población coreana residente en el oeste de Australia, los cuales guardaban una mayor relación con factores como el empleo, el estatus económico, nivel educativo, o el género (femenino), siendo este último probablemente el más significativo. Se observó también que la práctica de actividad física de la población coreana era significativamente más pequeña que la de la población autóctona y que sus actividades de ocio y recreación no guardaban relación con la de la población local australiana.

En relación a la Legislación sobre deporte e inmigración, se encontraron varios artículos relacionados con la regulación del mercado laboral deportivo por parte de los organismos legislativos de la Unión Europea. 
Así Gil (2002) y Maguirre y Pearton (2000) analizan lo que ha significado para el fútbol español y el mercado de fichajes internaciones la sentencia "Bosman". Esta sentencia supuso la aparición de dos categorías de jugadores de fútbol, los comunitarios de la Unión Europea y los no comunitarios. Así se estableció que los comunitarios no serían denominados dentro del territorio de la Unión como extranjeros, lo cual tuvo repercusiones directas en el aumento de jugadores europeos en las ligas de fútbol europeas (Alemania, Francia, Inglaterra y España) frente al resto de países del mundo, observándose como a veces los órganos legislativos europeos se ven obligados a pronunciarse para resolver problemas indirectos en el terreno deportivo.

\section{CONCLUSIONES}

- Los estudios sobre deporte profesional y migración de los talentos deportivos así como los estudios sociológicos relacionados con el deporte y la inmigración fueron los más numerosos.

- Los estudios de género e inmigración encontrados fueron fundamentalmente femeninos.

- El fútbol fue el deporte más nombrado en los estudios encontrados con 20 artículos, hallándose los programas de actividad física de mantenimiento en segundo lugar con siete.

- Por continentes, Europa y América del Norte, fueron los lugares de destino de las personas inmigrantes donde más estudios se han publicado, y como lugares de procedencia: América del Sur, Asia (sobre todo China e India) y África.

- La actividad física y el deporte fueron utilizados como medios de apoyo en la mayoría de los estudios encontrados y no como un fin en sí mismo, el nexo común en la mayoría de ellos fue la integración social. 


\section{LIMITACIONES DEL ESTUDIO}

Se quiere poner de evidencia que la revisión sistemática de estudios finalizó el tres de noviembre de 2011 por lo que todos los trabajos publicados con posterioridad no han podido ser incluidos en este documento.

Finalmente somos conscientes de que en general los trabajos analizados comparten entre ellos diferentes contenidos que los hacen muy heterogéneos y difíciles de clasificar, con lo que ha sido el criterio personal de los investigadores el establecer las seis áreas temáticas en las que finalmente se ha dividido el presente artículo. 
Analysis of the literature on immigrants, physical activity, sport and integration in English language

Abstract: The article discussed, is a review of the existing literature on social integration, immigrant groups, physical activity and sport. The literature found was obtained from international databases using various combinations of descriptors. All studies were classified with three criteria chronology, language and areas. From the information obtained in 50 articles it is concluded that the scientific field of sport and immigration is very heterogeneous, being athletic talent migration and sociological studies related to sport and immigration, the most repeated themes.

Keywords: Emigrants and Immigrants. Social values.Sports. Review.

\section{REFERENCIAS}

AGERGAARD, S. Elite athletes as migrants in Danish women's handball. International Review for the Sociology of Sport, London, v. 43, n. 1, p. 5-19, mar. 2008.

ALISON, D.; TRAYLOR, T. Sport and Physical Recreation in the Settlement of Immigrant Youth. Journal of the Canadian Association for Leisure Studies, Toronto, v. 31, n. 1, p. 27-29. 2007 
ARNAUD, L. Sport as a cultural system: Sports policies and (new) ethnicities in Lyon and Birmingham. International Journal of Urban and Regional Research, Hoboken, v 26, n 3, p. 571-587, 2002.

BLECKING, D. Sport and Immigration in Germany. The International Journal of the History of Sport, London, v. 25, n. 8, p. 955-973, 2008.

CAMERON, Y. The recruitment of New Zealand student athletes to the United States: A case study in sport migration. New Zealand Sociology, Auckland, v. 15, n. 2, 226-250, jun. 2000.

CARTER, T.F. Family networks, state interventions and the experience of Cuban transnational sport migration. International Review for the Sociology of Sport, London, v. 42, n. 4, p. 371-389, dic. 2007.

CHIBA, N. Pacific professional baseball leagues and migratory patterns and trends: 1995-1999. Journal of Sport \& Social Issues, London, v. 28, n. 2, 193-211, mayo 2004.

CORNELISSEN, S.; SOLBERG, E. Sport mobility and circuits of power: The dynamics of football migration in Africa and the 2010 World Cup. Politikon. Stellenbosch, v. 34, n. 3, p. 295-314, 2007.

CRONIN, M.; DOYLE, D.; O'CALLAGHAN, L. Foreign Fields and Foreigners on the Field: Irish Sport, Inclusion and Assimilation. The International Journal of the History of Sport, London, v. 25, n. 8, p. 1010-1030, jun. 2008.

DARVY, P.; ARKINDES, G.; KIRWIS, M. Football Academies and the Migration of African Football Labor to Europe. Journal of Sport \& Social Issues, London, v. 31, n. 2, p. 143-161, mayo 2007.

DIETSCHY, P. Football players' migrations: A political stake. Historical Social Research, Zurich, v. 31, n. 1, p. 31-41, 2006.

DIMEO, P.; VASCONCELLOS, C.H. 'I Am Not A Foreigner Anymore': a microsociological study of the experiences of brazilian futsal players in european leagues. Movimento, Porto Alegre, v. 15, n. 2, p. 33-44, feb. 2009.

EUN-OK E.-O, I.; MYOUNG-AE, C. Physical activity of Korean immigrant women in the U.S.: Needs and attitudes. International Journal of nursing studies, Milwaukee, v. 38, n. 5, p. 567-577, oct. 2001.

FALCOUS, M.; MAGUIRRE, J. Globetrotters and Local Heroes? Labor Migration, Basketball, and Local Identities. Sociology of Sport Journal, Toronto, v. 22, n. 2, p, 137-157, jun. 2005.

FLEMING, T. 'Now the African reigns supreme': The rise of African boxing on the Witwatersrand, 1924-1959. The International Journal of the History of Sport, London, v. 28, n. 1, p. 47-62, 2011. 
FROIDURE, C.; RAVENEL, L. Migration of professional basketball players in Europe. Mappemonde, Avignon, v. 98, n.2, feb. 2010. Disponible en: http:// mappemonde.mgm.fr/num26/articles/art10203.html. Acceso en: 20 mayo 2012.

GIL, S. Soccer and Migration. The Bosman Ruling in the Process of the Construction of the European Community (Chronicles from Spain). Migraciones Internacionales, Tijuana, v. 1, n. 3, p. 54-78, 2002.

HIBBINS, R. Sexuality and Constructions of Gender Identity among Chinese male Migrants in Australia, Asian Studies Review, London, v. 30, n. 3, p. 289 - 303, sept. 2006

HOSPER, K.; KLAZINGA, N.; STRONKS, K. Acculturation does not necessarily lead to increased physical activity during leisure time: a cross-sectional study among Turkish young people in the Netherlands. BMC Public Health, Amsterdan, v. 7, n. 9, p. 230 , sept. 2007.

HUMPHREYS, B.; MUNICH, D. Sport participation and migration. International Journal of Sport Management and Marketing, Hamburg, v. 3, n. 4, p. 335347, 2008.

LENZ, B. United in association? Municipal recreation and the integration of Polish and Masurian immigrants in the Ruhr area between 1900 and 1939. Archiv fur Sozialgeschichte, Bonn, n. 46, p. 183-203, 2006.

LOPES, M.; WADDINTON, I. Sport and globalization: the migration of Eastern European players to the Portuguese professional soccer league. Journal of Sports Sciences, London, v. 22, n. 6, p 580-589, jun. 2004.

MAGUIRRE, J. 'Real politic' or 'ethically based': Sport, globalization, migration and nation-state policies. Sport in Society, London, v. 11, n. 4, p. 443-459, jun. 2008.

MAGUIRRE, J. Sport labor migration research revisited. Journal of Sport \& Social Issues, London, v.28, n. 4, p. 477-482, nov. 2004.

MAGUIRRE, J.; PEARTON, R. The impact of elite labour migration on the identification, selection and development of European soccer players. Journal of Sports Sciences, London, v. 18, n. 9, p. 759-769, sept. 2000.

MARLIÈRE, E. Youth of suburbs territories and cultural practices. Ethnologie Francaise, Paris, v. 38, n. 4, p. 711-721, 2008.

MONIZ, M. Adaptive Transnational Identity and the Selling of Soccer: The New England Revolution and Lusophone Migrant Populations. Soccer \& Society, London, v. 8, n. 4, p. 457-477, ago. 2007.

MÜLER, F.; VANZOONEN, L.; DE ROODE, L. The Integrative Power of Sport: Imagined and Real Effects of Sport Events on Multicultural Integration. Sociology of Sport Journal, Toronto, v. 25, n. 3, p. 387-402, sept. 2008. 
PFISTER, G. Doing Sport in a Headscarft? German Sport and Turkish Females. Journal of Sport History, California, v. 3, n. 27, p. 497-524, 2000.

POLI, R. African migrants in Asian and European football: hopes and realities. Sport in Society, London, v.13, n. 6, p. 1001-1012, jul. $2010 \mathrm{~b}$.

POLI, R. Migration of football players and globalisation: From the world system to social networks. Mappemonde, Avignon, v. 88, n. 4, abr. 2007. Disponible en: http://mappemonde.mgm.fr/num16/articles/art07401.html. Acceso en: 20 mayo 2012.

POLI, R. Migrations and trade of african football players: Historic, geographical and cultural aspects. Africa Spectrum, Hamburgo, v. 41, n.3, p. 393-414, 2006.

POLI, R. Understanding globalization through football: The new international division of labour, migratory channels and transnational trade circuits. International Review for the Sociology of Sport, London, v. 45, n. 4, p. 491-506, dic. 2010a.

SCHOONDERWOERD, P. Shall We Sing a Song for You? Mediation, Migration and Identity in Football Chants and Fandom. Soccer \& Society, London, v. 12, n. 1, p. 120-41, ene. 2011.

SINE, A.; SORENSEN, J. Policy, sport and integration: the case of talented ethnic minority players in Danish football clubs. International Journal of Sport Policy, London, v. 2, n. 2, p. 205-222, jul. 2010.

SUGDEN, J. The challenge of using a values-based approach to coaching sport and community relations in multi-cultural settings. The case of Football for Peace (F4P) in Israel. European Journal for Sport and Society, Munster, v.3, n. 1, p. 724, ene. 2006.

TAINSKY, S.; STODOLSKA M. Population migration and team loyalty in professional sports. Social Science Quarterly, Champaign, v.91, n. 3, p. 801-15, jul. 2010.

TAKANASI, Y.; HOME, J. Moving with the Bat and the Ball Preliminary Reflections on the Migration of Japanese Baseball Labour. International Review for the Sociology of Sport, London, v.41, n. 1, p. 79-88, mar. 2006.

TAYLOR, M. Global players? Football, migration and globalization, c.1930-2000. Historical Social Research, Zurich, v. 31, n. 1, p. 7-10, 2006.

TCHA, S.S.; LOBO, F. Analysis of constraints to sport and leisure participation - the case of Korean immigrants in Western Australia. World Leisure Journal, London, v. 45 , n. 3 , p. $13-23,2003$.

TERRET, T. Introduction: Sport, Europe and Migration. The International Journal of the History of Sport, London, v. 25, n. 8, p. 953-954, 2008.

URNIAZ, J. Geopolitical and Cultural Characteristics of the Kaliningrad Province and Prospects of Social Integration through Sport with the Warmia-Mazury Region. Research Yearbook, Warszawa, v. 12, n. 2, p. 295-299, 2006. 


\section{ArtigosOrignais}

VASCONCELLOS, C.H.; DIMEO, P. The experience of migration for Brazilian football players. Sport in Society, London, v. 12, n.6, p. 725-736, ago. 2009.

YANG, X. Dance and Identity: The Chinese Immigrant Women's Leisure Activity in Vancouver. Journal of the International Council of Sport Science \& Physical

Education, Berlin, v. 50, p. 11-14, may. 2007.

Dirección postal:

Department of Physical Education and Sports

Faculty of Educational Sciences. Office 5.22

University of Seville

C/ Pirotécnia s/n

41013. Sevilla. Spain

Recebido em: 21-09-2012

Aprovado em: 04-11-2012

Movimento, Porto Alegre, v. 19, n. 01, p. 183-202, jan/mar de 2013. 